\title{
PHOTOSPHERIC EMISSION FROM GAMMA-RAY BURSTS
}

\author{
M. Axelsson ${ }^{1,2}$
}

\begin{abstract}
In spite of extensive research over the past decades, a complete physical picture of the origin of the prompt gamma-ray burst emission is still lacking. During recent years, evidence has been accumulating that the jet photosphere plays an important role. In this paper we summarize the lessons learned from Fermi observations regarding the behavior of the photosphere and discuss why photospheric emission does not necessarily appear as blackbody radiation. We concentrate on two strong and important bursts, GRB 090902B and GRB 110721A, which serve as examples of the standard appearance photospheric emission may have in gamma-ray burst spectra.
\end{abstract}

\section{Introduction}

Although the emission mechanisms active in the prompt phase of gamma-ray bursts (GRBs) are still under debate, there is much evidence that the photosphere of the relativistic outflow plays an important role in the formation of the observed spectrum (e.g. Lazzati \& Begelman 2010; Ryde et al. 2010; Guiriec et al. 2011; Vurm et al. 2011; Giannios 2011; Zhang et al. 2011). A strong contribution from the photosphere was predicted on physical grounds already in early works by Goodman (1986) and Paczyński (1986). However, the observed spectra are in general nonthermal so this was not considered a viable model. Here, we summarize observations of GRBs made with the Fermi Gamma-ray Space Telescope which suggest that photospheric emission is indeed present in the spectra of many GRBs.

\section{Early observations}

The first observational hints of a photospheric component in GRBs came from Compton Gamma-Ray Observatory/BATSE data (20-2000 keV). Ryde (2004)

\footnotetext{
1 Oskar Klein Center, Department of Physics, Stockholm University, 10691 Stockholm, Sweden

${ }^{2}$ Department of Physics, KTH Royal Institute of Technology, 10691 Stockholm, Sweden
} 

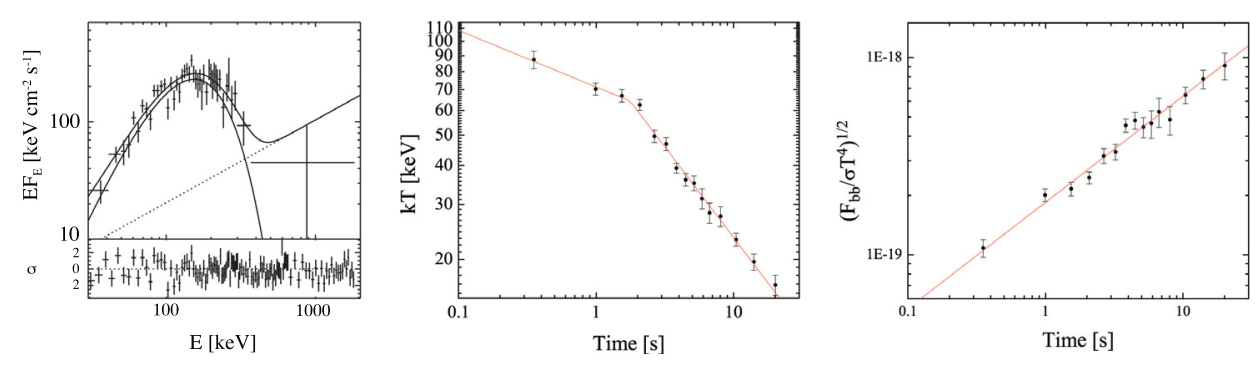

Fig. 1. Observational results from BATSE trigger 907. Left: spectrum showing a fit using a Planck function and power-law. Middle: evolution of the temperature of the Planck function during the burst. Right: ratio of observed flux to emergent flux.

found that in many individual emission pulses an equally good or better fit could be found by using a model comprising a Planck function and a power-law, as compared to the traditional Band function. Additionally, it was found that the evolution of the Planck function component during the prompt phase followed well defined and consistent characteristics. An example of such a BATSE observation is shown in Figure 1. The Planck component was interpreted as the photosphere of the GRB.

\section{Results from Fermi}

Since its launch in 2008, Fermi has seen over 1000 GRBs. The major advantage of Fermi over BATSE is that it has a much wider spectral coverage. Combining the two instruments - the Gamma-ray Burst Monitor (GBM; Meegan et al. 2009) and the Large Area Telescope (LAT; Atwood et al. 2009) - Fermi achieves continuous coverage from $8 \mathrm{keV}$ to above $300 \mathrm{GeV}$. Although the majority of the GRBs detected by Fermi are seen only in the low-energy range, there have been 35 bursts with high-energy emission strong enough to be detected also by the LAT.

\subsection{Multiple spectral components}

Ryde (2004) showed that an alternative fit of a Planck function plus power-law could be used instead of a Band function in many cases, over the limited energy range provided by BATSE. With the wider energy coverage of Fermi, the picture has matured. In several GRBs, an additional spectral component is found below the peak of the Band function (the low-energy region of the Band function corresponds to the power-law seen in the BATSE range). Examples of such bursts are GRB 090820A (Burgess et al. 2011) and GRB 100924B (Guiriec et al. 2011). Adding a Planck function at lower energies, in addition to the Band function covering the majority of the emission, significantly improves the fit.

Recently, results from analysis of Fermi data from GRB 110721A were published (Axelsson et al. 2012). They show that also in this burst there is a need for 

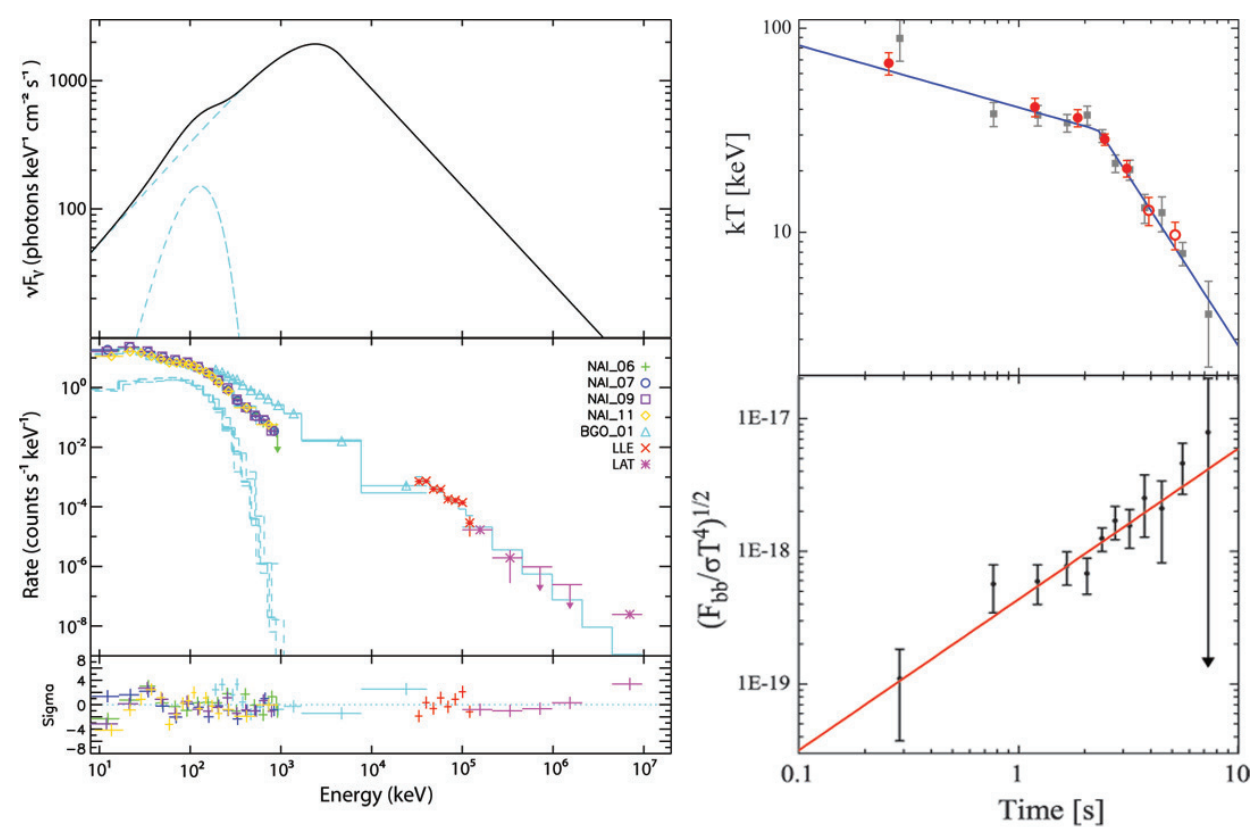

Fig. 2. Left: time-integrated spectrum of GRB 110721A fit with a model comprising a Band function combined with a Planck function. The upper panel shows the model spectrum in $\nu F_{\nu}$ representation, the middle panel the count spectrum and the lower panel the residuals of the fit. Right: evolution of the temperature (top) and ratio of observed to emergent flux (bottom) of the blackbody. In the top panel, filled circles indicate a $>5 \sigma$ significance of the blackbody, filled circles a $3 \sigma$ significance. The smaller points in both panels are from fits using a high time resolution, which lowers the significance of the component.

an extra component at low energies. During the first 3 seconds, this extra component (modeled with a Planck function) has a significance above $5 \sigma$. After this the significance drops to $3 \sigma$. The component is also required in the time-integrated spectrum, as shown in Figure 2.

In order to understand the nature of this extra component, we can study its evolution in time. Doing so, we find that it follows the same pattern as previously seen for the Planck function components in the BATSE observations (right panels in Figs. 1 and 2).

The fact that the components follow the same behavior strongly indicates that they are the result of the same physical process, and we connect them to the photosphere.

\subsection{Dominating photospheric emission}

Although the photospheric component is usually weak compared to the main emission peak fit by the Band function, in GRB 090902B the photospheric emission 
dominates. At early times, the spectrum of this GRB shows a narrow peaked component, as well as a separate power-law component observed at energies both above and below the peak (Abdo et al. 2009).

During the first half of the GRB emission pulse, the peaked component is very narrow, with $\alpha \sim 0.3$ and $\beta \sim-4$, thus strongly violating the optically thin synchrotron limit. This led Ryde et al. (2010) to identify the component with photospheric emission, and it was shown that a multicolor blackbody provides a good fit to the data. A multicolor blackbody is expected on theoretical grounds, and while a standard blackbody provides an adequate approximation when the component is weak, it is not sufficient when the component dominates. This clear identification also led to a unique possibility to study the evolution of the photospheric emission. It was found that during the later half of the burst, the peaked component broadened significantly, to resemble more the typical Band function peak seen in the majority of bursts.

As we are clearly observing the same dominant component throughout the duration of GRB 090902B, the drastic change of appearance that is seen is strong evidence that photospheric emission need not appear as blackbody emission. At early stages the photospheric component was indeed close to a blackbody, allowing its identification. However, during the event physical conditions in the outflow changed, and this was mirrored in the shape of the photospheric component.

\section{Non-Planckian photospheres}

As shown by GRB 090902B, photospheric emission is not synonymous with blackbody radiation. The questions then arises on how the different spectral shapes can arise.

One possibility that is being explored is subphotospheric emission. In brief, in this scenario energy is dissipated below the photosphere, modifying the emergent spectrum. Different models propose different origins, such as magnetic reconnection (Giannios 2008), internal shocks (Ioka 2010) or collisional dissipation (Beloborodov 2010). By varying the amount of dissipation and parameters of the outflow, it is possible to produce a wide range of spectral shapes by such subphotospheric energy release (Pe'er et al. 2006; Nymark et al. 2011).

Recently, Lundman et al. (2012) showed that purely geometrical effects will also produce a broadening of the emergent spectrum. Considering relativistic limb darkening, they use a combination of analytical model and Monte Carlo simulation to study the emergent spectrum from a jet. They find that for a narrow jet, where the opening angle is of the order of the relativistic beaming angle, a broadening of the photospheric spectrum is expected for any viewing angle. For a broader jet, the broadening effect is strong only if the viewing angle lies along the edge of the outflow, i.e. is close to the jet angle.

\section{Summary}

The observations of GRBs with Fermi support previous claims of photospheric emission from e.g. BATSE. A growing number of GRBs show spectra with more 
than one peaked component, and a Band function alone is not sufficient to model them. When adding a second component, the fit improves significantly. This second component follows well-defined characteristics, and we interpret it as due to photospheric emission. The bright GRB 090902B clearly shows that photospheric emission does not necessitate a Planck function in the spectrum, which is also supported by theoretical considerations. Several broadening mechanisms exist which will modify the emergent spectrum, e.g. subphotospheric dissipation and geometrical broadening. Including a photospheric component is thus a first step to understanding the physical origin of GRB prompt emission - something which the Band function cannot provide.

The Fermi LAT Collaboration acknowledges support from a number of agencies and institutes for both development and the operation of the LAT as well as scientific data analysis. These include NASA and DOE in the United States, CEA/Irfu and IN2P3/CNRS in France, ASI and INFN in Italy, MEXT, KEK, and JAXA in Japan, and the K.A. Wallenberg Foundation, the Swedish Research Council and the National Space Board in Sweden. Additional support from INAF in Italy and CNES in France for science analysis during the operations phase is also gratefully acknowledged.

\section{References}

Abdo, A.A., Ackermann, M., Ajello, M., et al., 2009, ApJ, 706, L138

Atwood, W.B., Abdo, A.A., Ackermann, M., et al., 2009, ApJ, 697, 1071

Axelsson, M., Baldinid, L., Barbiellini, G., et al., 2012, ApJ, 757, L31

Beloborodov, A., 2010, MNRAS, 407, 1033

Burgess, J.M., Preece, R.D., Baring, M.G., et al., 2011, ApJ, 741, 24

Giannios, D., 2011 [arXiv: 1111.4258]

Giannios, D., 2008, A\&A, 480, 305

Goodman, J., 1986, ApJ, 308, L47

Guiriec, S., Connaughton, V., Briggs, M., et al., 2011, ApJ, 727, L33

Ioka, K., 2010, Progr. Theor. Phys., 124, 667

Lazzati, D., \& Begelman, M.C., 2010, ApJ, 725, 1137

Lundman, C., Pe'er, A., \& Ryde, F., 2012 [arXiv: 1208.2965]

Meegan, C., Lichti, G., Bhat, P.N., et al., 2009, ApJ, 702, 791

Nymark, T., Axelsson, M., Lundman, C., et al., 2011 [arXiv:1111.0308]

Paczyński, B., 1986, ApJ, 308, L43

Pe'er, A., Mészáros, P., \& Rees, M.J., 2006, ApJ, 642, 995

Ryde, F., 2004, ApJ, 614, 827

Ryde, F., Axelsson, M., Zhang, B.-B., et al., 2010, ApJ, 709, L172

Vurm, I., Beloborodov, A.M., \& Poutanen, J., 2011, ApJ, 738, 77

Zhang, B.-B., Zhang, B., Liang, E.-W., et al., 2011, ApJ, 730, 141 
